\title{
Accuracy, discriminative properties and reliability of a human ESC-based in vitro toxicity assay to distinguish teratogens responsible for neural tube defects
}

\author{
Anne-Catherine Feutz ${ }^{1,2,3} \cdot$ Christian De Geyter ${ }^{1,3}$
}

Received: 28 February 2019 / Accepted: 4 July 2019 / Published online: 10 August 2019

(c) The Author(s) 2019

\begin{abstract}
The poor correlation of developmental toxicity studies in animals with human outcome data has emphasized the need for complementary assays based on human cells and tissues. As neural tube defects represent an important proportion of congenital malformations, we evaluated here the accuracy of a human embryonic stem cell (hESC)-based assay to predict chemically induced disruption of neural tube formation. As teratogenic compounds, we used cyclopamine (CPA), valproic acid (VPA), ochratoxin A (OTA) and mycophenolic acid (MMF), all suspected or known inducers of human neural tube defects, as well as theophylline and saccharin as negative control compounds. We analyzed their effects on the ability of hES cells to give rise to neural precursors (expressing specific marker Nestin), to form neural tube-like structures (rosettes), and to express specific markers (Sox1, Otx2, Lix1, EvI1, Rspo3) during rosette formation. The results showed that various effects of the selected compounds on early neural development could be specifically revealed in vitro through related alterations of neurogenic differentiation of hESC. Furthermore, it was possible to discriminate toxicants acting at different time points during embryonic development and, therefore, responsible for distinct adverse effects on neural tube formation. By comparing four different hESC lines, we observed a significant (up to fivefold) variability of the line-dependent response to toxicants. We highlight at least two sources of variability: one related to the heterogeneity of hESC lines in culture (stemness/commitment profiles); the second to possible genetically determined differences in individual sensitivity to teratogens.
\end{abstract}

Keywords Developmental neurotoxicity $\cdot$ Neural tube defect $\cdot$ Human embryonic stem cells $\cdot$ In vitro test

$\begin{array}{ll}\text { Abbreviations } \\ \text { CPA } & \text { Cyclopamine } \\ \text { hESC } & \text { Human embryonic stem cell } \\ \text { MMF } & \text { Mycophenolate mofetil } \\ \text { NTD } & \text { Neural tube defect }\end{array}$

Electronic supplementary material The online version of this article (https://doi.org/10.1007/s00204-019-02512-8) contains supplementary material, which is available to authorized users.

Christian De Geyter

Christian.DeGeyter@usb.ch

1 Reproductive Medicine and Gynaecological Endocrinology (RME), University Hospital, University of Basel,

Vogesenstrasse 134, 4031 Basel, Switzerland

2 Department of Biomedicine (DBM), University Hospital, University of Basel, Hebelstrasse 20, 4031 Basel, Switzerland

3 SCAHT - Swiss Centre for Applied Human Toxicology, University of Basel, Missionsstrasse 64, 4055 Basel, Switzerland

\section{OTA Ochratoxin A}

VPA Valproic acid

\section{Introduction}

Major birth defects affect $2-8 \%$ of all newborn children (reviewed by Eurocat, 2005; Queisser-Luft et al. 2002). A large proportion of congenital abnormalities consist of neurodevelopmental defects, most importantly disruption of neural tube formation and closure (reviewed in Copp and Greene 2013). A number of toxicants have been demonstrated to exert harmful effects on neuro-development (Grandjean and Landrigan 2006). Currently, routine assessment in neurotoxicology requires extensive animal experimentation (OECD TG426 2007), but discordant effects among species and the urge to reduce animal experimentation have emphasized the need for the development of in vitro human assays (Gassmann et al. 2010; Harrill et al. 
2011; Leist and Hartung 2013; Seok et al. 2013; Baumann et al. 2016).

In recent years, efforts have been invested in the development of complementary assays based on human embryonic stem cells (hESC) or human-induced pluripotent cells (hiPS). As neural differentiation of embryonic stem cells in vitro recapitulates different phases of early embryonic development (Kelava and Lancaster 2016), a system based on stem cells directed to early neural differentiation may thus be used for testing purposes in toxicology. Some studies have already been conducted, focusing on cell proliferation, apoptosis, differentiation into various neural cell types, neurite outgrowth, cell migration and synaptogenesis (Colleoni et al. 2011; Harrill et al. 2011; Zimmer et al. 2012, 2014), on formation of neural tube-like structures called rosettes (Stummann et al. 2009; Colleoni et al. 2012; Talens-Visconti et al. 2011; Shinde et al. 2015), and on changes in gene expression patterns (Balmer et al. 2012; Meganathan et al. 2012; Krug et al. 2013; Schulpen et al. 2015; Waldmann et al. 2017). While several convincing case studies have now been described for a small number of compounds, we still lack evidence about the accuracy and reliability of an in vitro assay based on stem cells for the prediction of toxicity. Therefore, such tests have not yet been introduced into routine toxicology (Bal-Price et al. 2018; Fritsche et al. 2018).

In the present study, we tested the feasibility of a hESCbased culture system for identifying the neurodevelopmental toxic effects of chemicals known, cyclopamine (CPA), valproic acid (VPA) and ochratoxin A (OTA), or suspected, mycophenolate mofetil (MMF), to alter the formation of the neural tube (Supplementary Table 1). We particularly focused on evaluating the assay's capability to distinguish and possibly predict different types of neural tube defects (NTDs). In addition, we evaluated the reliability of the assay using four different hESC lines, all established and characterized in the same laboratory under similar conditions (Sterthaus et al. 2014).

\section{Materials and methods}

\section{Maintenance, expansion and differentiation of human embryonic stem cells}

Establishment and characterization of hESC lines CHES2, CHES3, CHES5, and CHES6 were described by Sterthaus et al. (2014). They were maintained on a human feeder layer (35 Gy $\gamma$-irradiated foreskin fibroblasts; ATCC CRL2429) in hESC medium: KnockOut ${ }^{\mathrm{TM}}$ DMEM culture medium (Invitrogen, Nr. 10829-018) supplemented with 20\% KSR (Invitrogen Nr. 10828-028), 2 mM L-GlutaMax (Invitrogen Nr. 35050-038), 0.05 mM $\beta$-mercapto-ethanol (Invitrogen
Nr. 31350-010), $0.1 \mathrm{mM}$ non-essential amino acid (Invitrogen Nr. 11140-035), 8 ng/ml bFGF (R\&D System Nr. 234FSE-025/CF), Gibco ${ }^{\mathrm{TM}}$ Penicillin-Streptomycin $(100 \mathrm{U} / \mathrm{ml}$ Invitrogen Nr. 15140-122).

For expansion, hESC colonies were mechanically removed from the feeders, dissociated into small clumps and amplified in suspension in ultra-low attachment 24-well plates (Corning Costar) in mTeSR ${ }^{\mathrm{TM}} 1$ medium (STEMCELL technologies 05850). Cell clusters were partially dissociated once a week by gentle trituration and cultured in the presence of ROCK inhibitor $10 \mu \mathrm{M}$ (Sigma Y0503) the first day.

For neural differentiation, the clusters were gently dissociated and plated in polyornithine $0.01 \%$ (Sigma P4957) and laminin $10 \mu \mathrm{g} / \mathrm{ml}$ (Sigma L2020) precoated wells at a density of $80000 \mathrm{cell} / \mathrm{cm}^{2}$. They were cultured in neural differentiation medium consisting of DMEM/F12 (Invitrogen 31331028), $1 \times$ N-2 Supplement (Invitrogen 17502048), $0.1 \mathrm{mM}$ non-essential amino acids, Penicillin-Streptomycin $(100 \mathrm{U} / \mathrm{ml})$ and recombinant human noggin $600 \mathrm{ng} / \mathrm{ml}$ (Prospec CYT-475) for 8 days.

\section{Selection of colony subpopulations}

The selection procedure is described in the legend of supplementary Figure S1. Selected colonies were cultured and amplified as described above. Forty colonies were analyzed by qRT-PCR, as described below.

\section{Exposure to toxicants}

The cells were exposed to the test compounds during neural commitment until the 8th day of differentiation, thus encompassing the acquisition of polarized contractile neuro-epithelial properties and the generation of neural precursors. Different concentrations of each chemical: theophylline (Sigma T1633) at 2, 6, 20, 60, 200, 600, 2000 and $6000 \mu \mathrm{M}$, saccharin sodium salt hydrate (Sigma S1002) at 0.002, 0.02, $0.2,2,20$ and $50 \mathrm{mM}$, cyclopamine hydrate (CPA; Sigma $\mathrm{C} 4116$ ) at $0.016,0.05,0.16,0.5,1.6,5$ and $16 \mu \mathrm{M}$, valproic acid sodium salt (VPA; Sigma P4543) at 0.08, 0.25, 0.8, 2.5 and $8 \mathrm{mM}$, ochratoxin A (OTA; Sigma 32937) at 0.5, 1, 2, 4, 10, 25, 62,5 and $125 \mathrm{nM}$ and mycophenolic acid (MMF; Sigma M3536) at 11.5, 23, 46, 92, 185 and $470 \mathrm{nM}$ were added at day 2 and day 5 after plating. At day 8, the rosettes were counted into each well under a Leica microscope at 10x magnification and the cells then harvested. For chemicals prepared into DMSO, the final DMSO concentration was $0.1 \%$ for all conditions including untreated samples. Experiments with CPA and VPA were performed on all 4 hESC lines, except cytotoxicity testing that was done with CHES6. Experiments with OTA and MMF were performed only on CHES6. All data presented are from three independent experiments, each performed in duplicate. 


\section{Quantification of cell survival by Alamar Blue assay}

Cells were plated at 125000 cells $/ \mathrm{cm}^{2}$ in 96 well-plates coated with polyornithine $0.01 \%$ (Sigma P4957) and laminin $10 \mu \mathrm{g} / \mathrm{ml}$ (Sigma L2020), cultured in neural differentiation medium and exposed to the compounds as described above. At day 8 , the cytotoxicity was assessed following the manufacturer's instructions. 10X ready-to-use AlamarBlue ${ }^{\circledR}$ reagent (Invitrogen DAL1025) was added to the cells in the culture medium followed by 5 -h incubation at $37{ }^{\circ} \mathrm{C}$. Finally, absorbance was measured with a spectrophotometer reader Synergy H1 (BioTek) at the wavelengths of $570 \mathrm{~nm}$ and $600 \mathrm{~nm}$.

\section{Quantification of relative gene expression levels by qRT-PCR}

Total RNA was isolated using the NucleoSpin RNA II kit (Macherey-Nagel 740955) according to the manufacturer's instructions. For each sample, $300 \mathrm{ng}$ of total RNA was reverse-transcribed into single-strand cDNA by the SuperScript ${ }^{\circledR}$ II Reverse Transcriptase (Invitrogen 18064.014) in the presence of $0.5 \mu \mathrm{M}$ of dNTP (Invitrogen 10297-018) and $5 \mathrm{ng} / \mu \mathrm{l}$ of random hexamer primers mix (Invitrogen 48190-011). 1/20 of the cDNAs was used for each PCR performed using Power SYBR ${ }^{\circledR}$ Green PCR Master mix (Applied Biosystems 4367659) in an ABI 7500 Fast Real-Time PCR System (Applied Biosystems). The different primers used were synthesized by Microsynth (Balgach, Switzerland) and are described in Supplementary Table 2.

\section{Results}

\section{Specificity of early neurodevelopmental changes after exposure to chemicals}

Dose-response experiments on $\mathrm{hES}$ cells differentiating into the neural lineage were carried out with the panel of six chemicals. The number of formed rosettes as well as the level of Sox 1 expression, a marker of mature rosettes coinciding with closure of the human neural tube in vivo, were determined. As rosette formation in culture depends on cell density, we also quantified the effects of the compounds on cell viability to distinguish any alteration of neural development caused by poor cell survival only.

High doses of every compound, including the negative control substances theophylline and saccharin, invariably induced cell death and any decrease in cell number was always accompanied by a decrease in the number of rosettes. Consequently, the dose-response curves for cell viability and rosette formation were perfectly superimposed in the absence of specific effect on neural development (Fig. 1a, b). However, this was also true for two of the three known neurodevelopmental toxicants, VPA and CPA, for which the two curves were indistinguishable (Fig. 1c, d). Thus, for those compounds, quantification of rosette number alone did not allow to discriminate between deleterious effects caused by biologically relevant or irrelevant decrease in cell viability in vitro. In contrast, OTA exerted a more drastic detrimental effect on rosette formation than on cell viability (Fig. 1f). The OTA concentration causing a 50\% reduction in the number of rosettes (EC50) was fourfold lower than that for a similar reduction of cell viability. In that case, some initial events involved in the formation of rosettes were thus clearly specifically affected independently of an effect on cell number.

In control cultures treated with theophylline or saccharin, the expression level of Sox 1 remained unaffected (Fig. 1a, b). This indicated that, once initiated and whatever their number, rosette development proceeded normally. In contrast, rosette maturation was strongly compromised in the presence of all three known NTD-inducing toxicants (Fig. 1c, d, f). Furthermore, the sensitivity of the process to each substance was different. In the presence of VPA, the decrease in Sox 1 expression followed a curve very similar to the reduction of rosette number. In the presence of CPA, Sox 1 expression was threefold less affected than rosette formation. In contrast, Sox 1 expression was 30-fold more affected by OTA and was almost abolished at a dose in which $60 \%$ of the normal number of rosettes was still observed and $90 \%$ of the cells still living.

For MMF, which is suspected to cause spina bifida (Fig. 1e), there was a clear effect on rosette maturation. Dose-response curves for Sox 1-expression and rosette number were very similar and thus the response to MMF was closest to that of VPA.

\section{Comparing the effects on maturation of neuro-epithelial structures and on generation of neural precursors}

During embryonic development, morphogenesis of the neural tube and the generation of neural precursors occur concomitantly. In vitro, however, neural precursors can be derived from ES cells without the formation of neuro-epithelial structures. We, therefore, determined whether gene expression quantification of the neural precursor marker, Nestin, was valuable as a complementary criterion to characterize NTD-inducing chemicals. We thus compared the expression levels of Nestin and Sox 1 in cells exposed to the six compounds (Fig. 2).

No effect on the expression levels of Nestin and Sox 1 was detected in the presence of the two control compounds (Fig. 2a, b). In contrast, Nestin expression was strongly reduced by exposure to all four neurodevelopmental toxicants. 
(a)

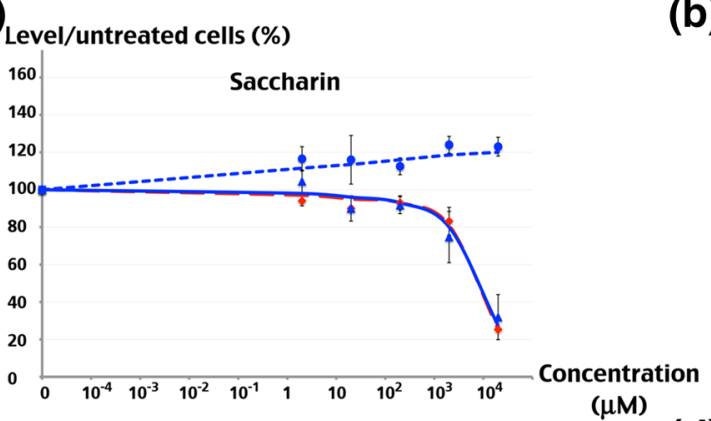

(c)

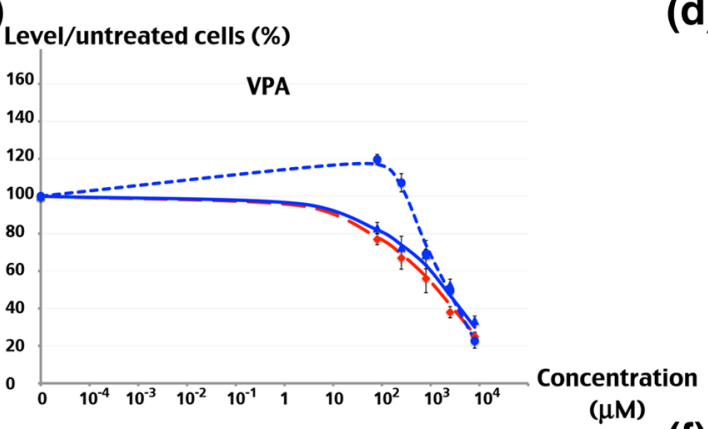

(e)

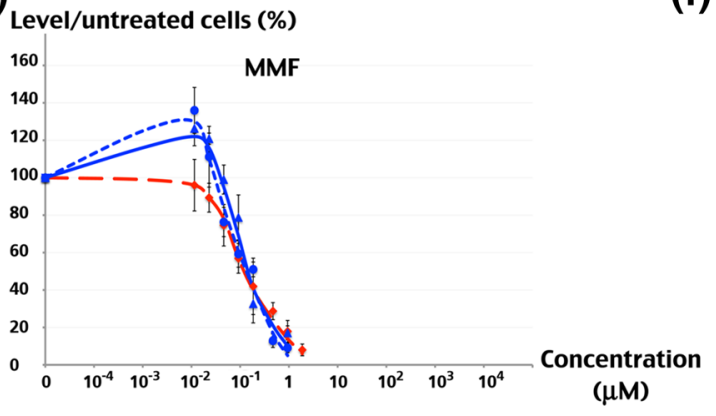

Fig. 1 Comparison of NTD-inducing toxicant effects on cell viability and rosette formation. CHES6 cells were exposed to various concentrations of chemicals from day 2 to day 8 and the different effects were quantified at day 8 . Dose-response curves for the number of formed rosettes, mRNA expression of the rosette marker Sox1 and

For three of them (VPA, CPA and MMF), the dose-response curves of both Nestin and Sox1 expression were very similar (Fig. 2c-e). The EC50 calculated for Nestin and Sox1 expression levels was 15 and $18 \mu \mathrm{M}$ with CPA, 90 and $100 \mathrm{nM}$ with $\mathrm{MMF}$, respectively, and $2 \mathrm{mM}$ with VPA for both genes. In contrast, the expression levels of Nestin and Sox 1 were differently affected by exposure to OTA (Fig. 2f), suggesting that rosette maturation was more affected than precursor generation. However, the OTA EC50s still differed only by a factor 2.5: $2 \mathrm{nM}$ for Nestin and $5 \mathrm{nM}$ for Sox1.

\section{Comparison of additional markers of rosette formation}

We hypothesized that disturbance during early neural induction might indirectly but globally impact the formation of (b)

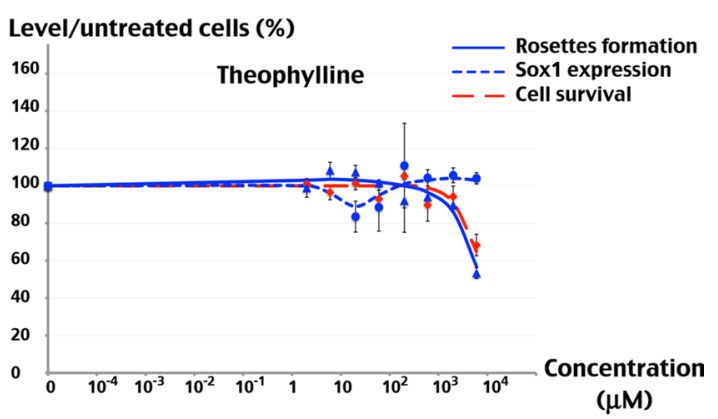

d)
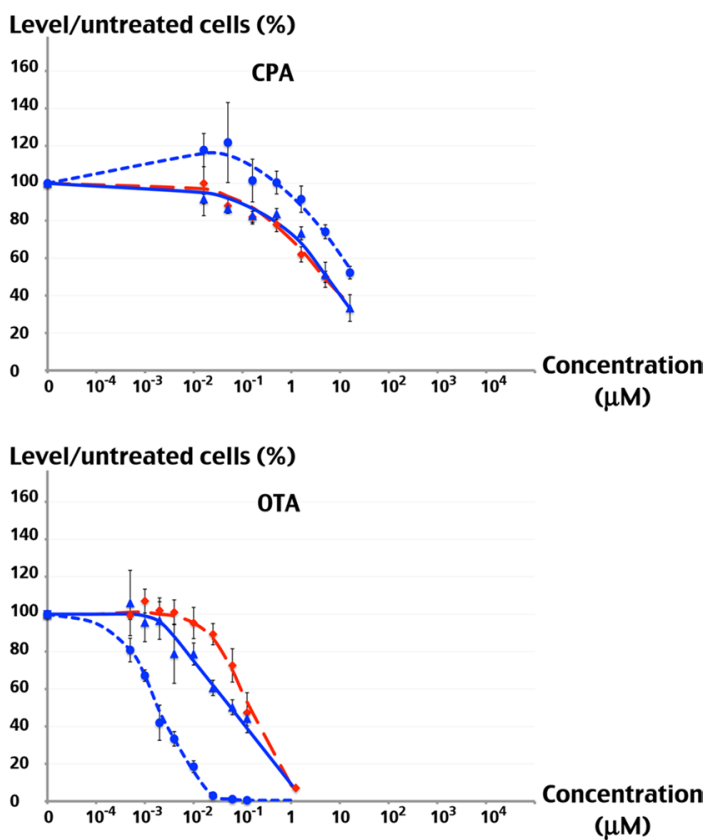

cell survival are shown for saccharin (a), theophylline (b), VPA (c), CPA (d), MMF (e) and OTA (f). All values are normalized relatively to untreated cells and the data are presented by their percentage (\%). Data are presented as mean \pm SEM of three independent experiments, each performed in duplicate

rosettes later on. If so, all gene products associated with the process of ongoing rosette formation would be affected similarly. In contrast, a compound directly affecting morphogenesis of the neural tube at the time of its formation would affect these genes differently depending on its mode of action. To test this hypothesis, in addition to the already chosen marker Sox1, we selected four additional genes, Otx2, Lix1, Evi1 and Rspo3, all strongly expressed in rosettes (Koch et al. 2009, Supplementary Fig. S1). We compared their respective sensitivity to the four NTD-inducing toxicants (Fig. 3).

Exposure to CPA (responsible for holoprosencephaly) reduced the expression of all markers very similarly, if not identically. Their dose-response curves largely overlapped (Fig. 3b) and the estimated EC50s did not differ by more than factor 1.3. In contrast, each marker responded 
(a)

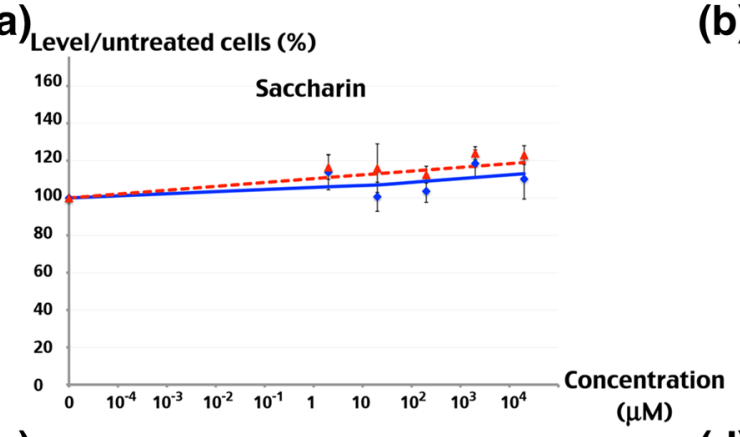

(c)

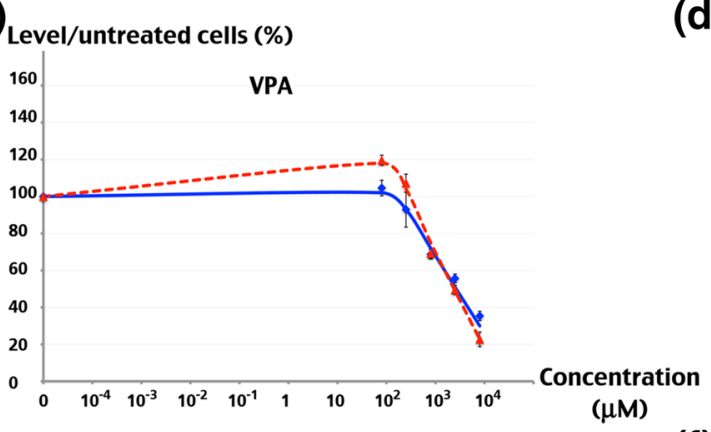

(e)

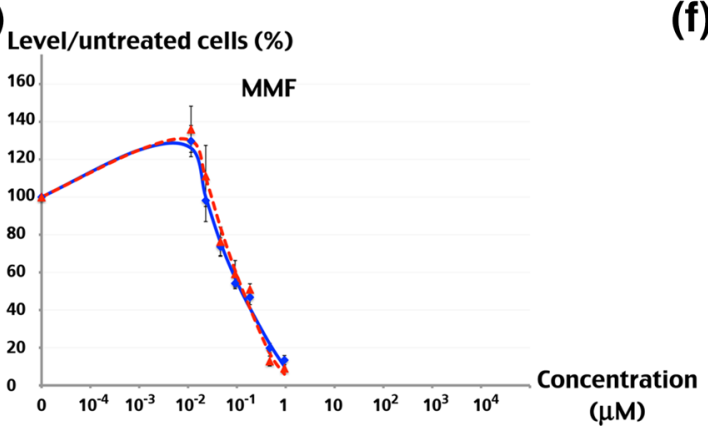

Fig. 2 Comparison of toxicants effects on morphogenetic (rosettes) and differentiation (neural precursors) markers. mRNA expression levels of Nestin and Sox 1 after exposure to toxicants were quantified

differentially to VPA (responsible for spina bifida) (Fig. 3a). For instance, the expression level of Otx2 was already less than half of its normal value at a dose for which the expression levels of Evi1 or Rspo3 remained unaffected. With VPA, we observed a tenfold sensitivity difference between the more (Otx2) and less affected gene (Lix1). Exposure to OTA, which is associated with either holoprosencephaly or exencephaly, led to an intermediate response profile (Fig. 3d). Dose-response curves were different but the difference in sensitivity between the more (Sox1/Lix1) and the less responsive markers (Evi1/Rspo3) was only threefold. Interestingly, VPA and MMF, both presumed responsible for spina bifida, exhibited the more comparable response profiles (Fig. 3a, c). After exposure to MMF, the markers were affected differentially and the sensitivity of the more (Otx2) and less affected (Evi1) markers differed by a factor 7 .

Notably, exposure to the three compounds thought to alter directly neural tube formation (VPA, MMF and OTA) (b)

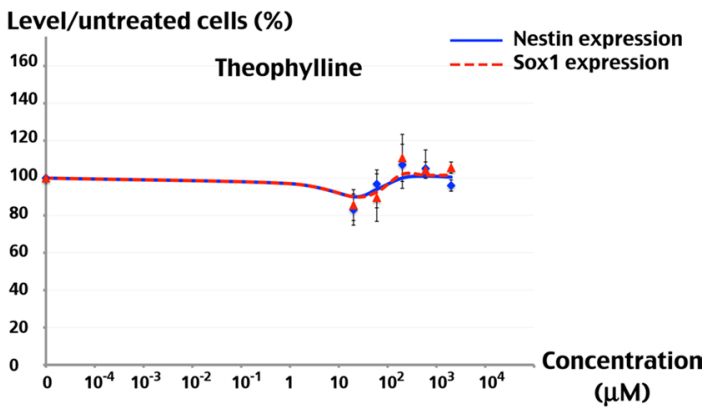

(d)

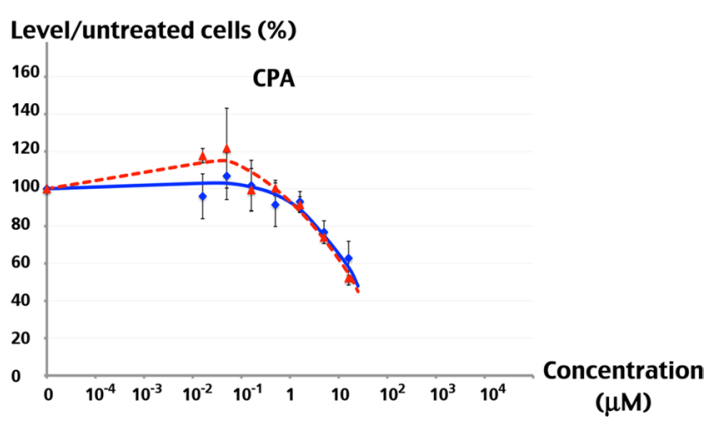

(f)

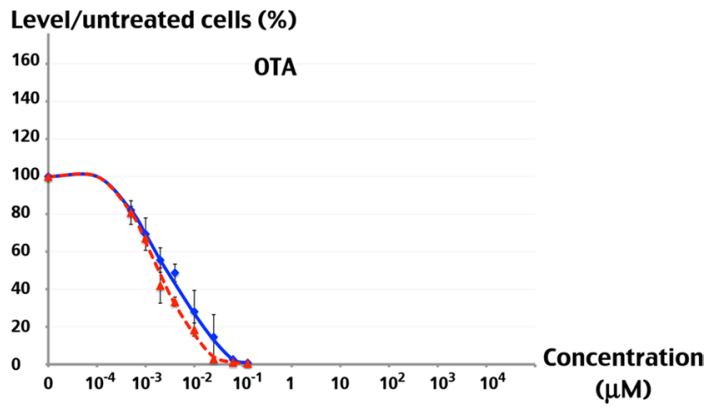

as in Fig. 1. Dose-response curves are shown for saccharin (a), theophylline (b), VPA (c), CPA (d), MMF (e) and OTA (f)

always affected Otx 2 and Sox 1 much more strongly than Evil and Rspo3 expression (Fig. 3a, c, d). That difference correlates with different onset of marker expression during the gradual process of rosette formation (Supplementary Fig. S1). Evi1 and Rspo3 are already slightly expressed in immature neuro-epithelium, therefore, before rosette formation (and possibly before action of those toxicants), and expression progressively increases to culminate in the mature rosette. In contrast, Otx 2 expression starts later on in more advanced, contracted and rosette-forming, neuro-epithelium and Sox 1 expression is restricted to polarized mature rosettes. Relatively to other rosette markers, expression of Lix 1 was differently affected by each toxicant. For instance, Lix 1 expression strongly decreased after exposure to MMF but showed the strongest resistance to VPA. These results likely reflect different periods and modes of action of the four compounds, which could thus be distinguished. 


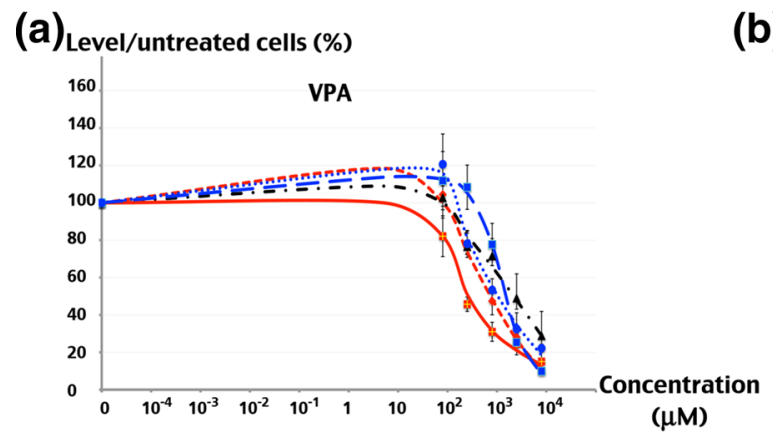

(c)

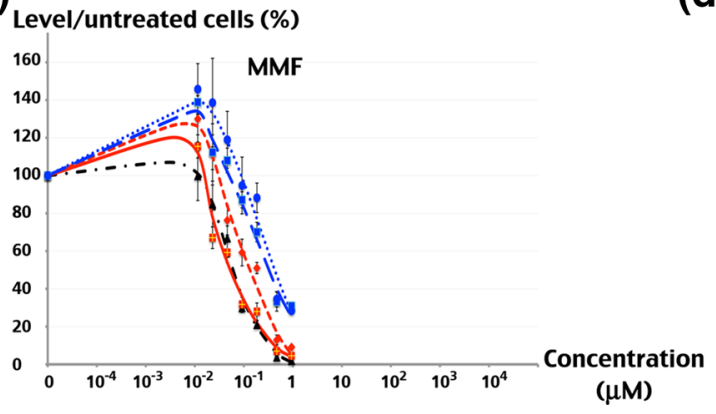

(b)

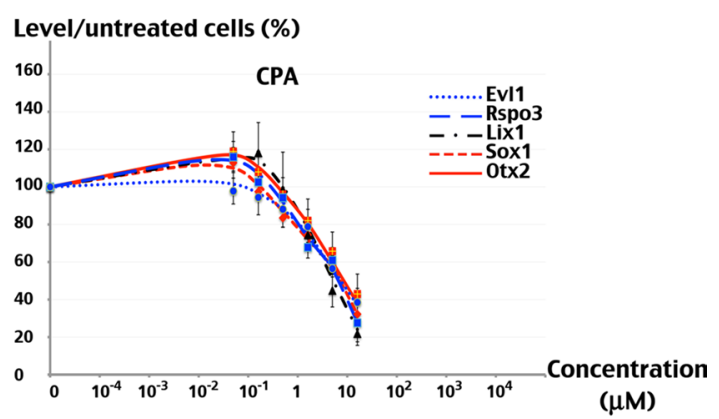

(d)

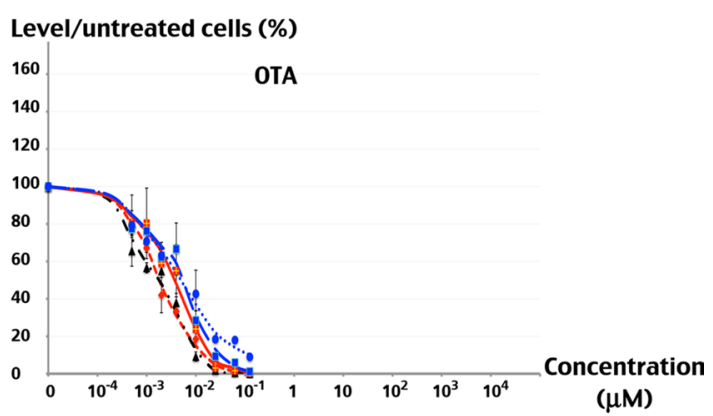

Fig. 3 Sensitivity of different rosette markers to NTD-inducing compounds. The effects of VPA (a), CPA (b), MMF (c) and OTA (d) on mRNA expression levels of Evi1, Rspo3, Lix1, Sox 1 and Otx2 were determined as described in Fig. 1

\section{Cell line-dependent variability of response to neurodevelopmental toxicants}

We compared the results obtained with three additional lines (CHES2, CHES3, CHES5) with those obtained with the previously used CHES6 cell line. Experiments were performed with one toxicant (CPA) acting early, during neural induction, and one (VPA) acting later, during neural tube morphogenesis. Their effects on the expression levels of both Nestin and Sox 1 were quantified in all $4 \mathrm{hESC}$ lines.

Each cell line responded differently to the toxicants (Fig. 4), resulting in a difference in the estimation of the toxic dose by up to a factor of 5 . The resistance/sensitivity status of a particular cell line varied depending on the compound tested and on the developmental process analyzed. For instance, Sox 1 expression in CHES2 was both more affected by CPA, but less affected by VPA, than in any other cell line (compare Fig. 4c and d). On the contrary, Sox 1 expression was less affected by CPA in CHES6 than in other lines, while it was very sensitive to exposure to VPA. The range of variation between cell lines was similar for the response to VPA or CPA when considering Sox 1 expression (around fivefold, Fig. 4c, d), but was different for Nestin expression (fivefold with CPA but less than twofold with VPA, Fig. 4a, b).

The effect of VPA on both Sox 1 and Nestin expression revealed the existence of two distinct groups of hESC lines (Fig. 4a, c). CHES3 and CHES6 were always more affected than CHES2 and CHES5. Within each group, the response to VPA was similar. This was particularly apparent for Nestin expression; dose-response curves for CHES3 and CHES6 on the one hand and CHES2 and CHES5 on the other hand were almost identical (Fig. 4a). Interestingly, those two groups correspond to a classification which we previously proposed based on gene expression profiles and differentiation behaviors (Sterthaus et al. 2014). For instance, CHES2 and CHES5 initially expressed similar levels of the stemness marker Oct4, approximately half of those expressed by CHES3 and CHES6. They also expressed similar levels of the neural commitment marker Pax6, approximately double those expressed in CHES3 and CHES6 (Fig. 5a).

As heterogeneity in hESC gene expression profiles relies on the coexistence of cell subpopulations expressing different levels of stemness and commitment markers (Cahan and Daley 2013), we isolated 48 different subpopulations of CHES6 and CHES2 cell lines (Supplementary Fig. S2). They were selected by their capacity to form colonies after dissociation to single cells, and cultured in highly diluted conditions. As illustrated for six of them (three derived from CHES6 and three from CHES2), it was thus possible to obtain subpopulations of both lines which exhibited similar stemness/commitment profiles, or subpopulations from the same line with different profiles of stemness/commitment, in order to compare their response to toxicants (Fig. 5b). However, those profiles turned out to be unstable. After an expansion phase, required to obtain the cell number needed to perform toxicity assays, they had reverted to profiles not 
(a)
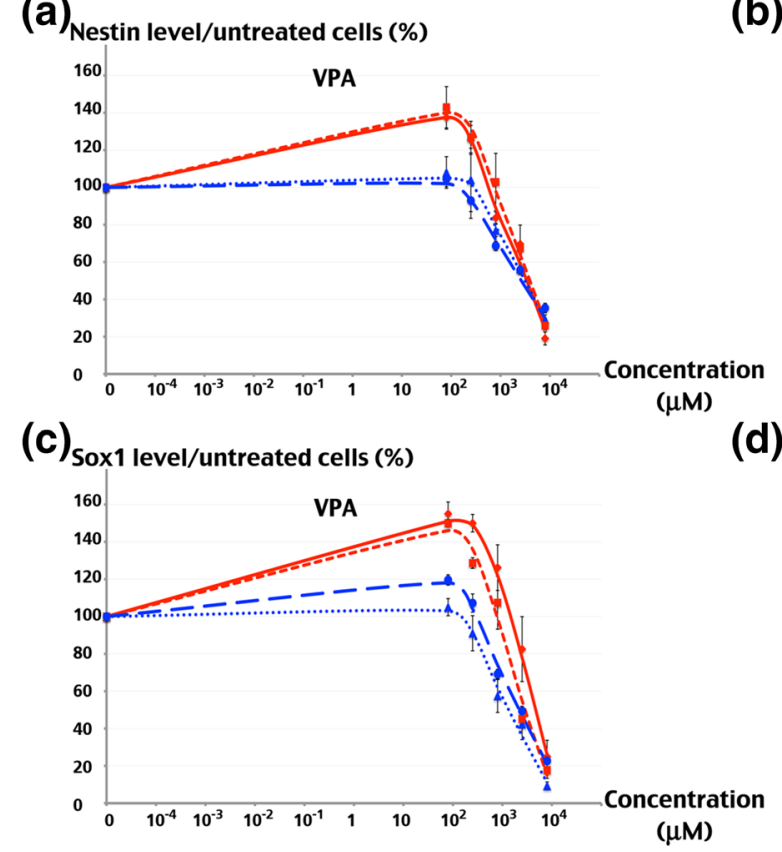

Fig. 4 Response of four different hESC lines to two toxicants acting at different developmental time points. Dose-response curves for mRNA expression level of Nestin $(\mathbf{a}, \mathbf{b})$ and $\operatorname{Sox} 1(\mathbf{c}, \mathbf{d})$ after expo-

significantly different from that of their mother cell lines (Fig. 5c).

For CPA, response variability reflected the different behavior of only one cell line whereas the response was similar among the three other cell lines (Fig. 4b, d). However, the eccentric line was different when considering rosettes maturation or precursors generation. Nestin expression decreased similarly after exposure to CPA in CHES3, CHES5 and CHES6, but was much strongly affected in CHES2 (Fig. 4b). The effect of CPA on Sox 1 expression in CHES2 was, in contrast, similar to CHES3 and CHES5 whereas CHES6 showed a higher resistance to the toxicant (Fig. 4d). Consequently, the use of multiple hESC lines demonstrated that rosette maturation was actually more affected by CPA than the generation of neural precursors, in contrast to the data obtained with CHES6 alone.

\section{Discussion}

We aimed to assess the accuracy and discriminative capacity of hESC-based assays to identify neurodevelopmental toxicity by characterizing the effects of three chemicals, VPA, CPA and OTA, known to induce different types of NTDs. We here demonstrate that the assay is able to both specifically identify early neurodevelopmental toxicity and to distinguish toxicants affecting different neurodevelopmental processes. We used their response profiles as references (b)

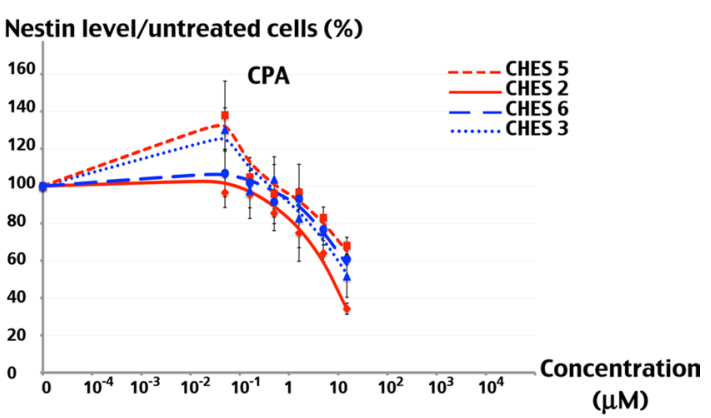

(d)

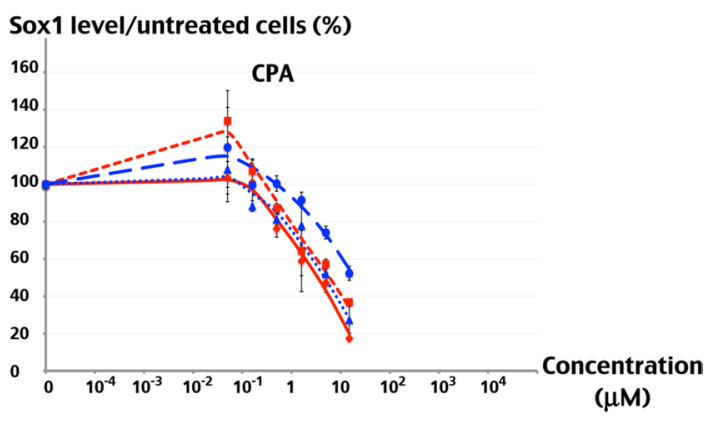

sure of CHES2, CHES3, CHES5 and CHES6 to VPA (a, c) and CPA (b, d) were determined as described in Fig. 1

to confirm that MMF, a suspected candidate for induction of spina bifida, is very likely a neurodevelopmental toxicant responsible for defects in neural tube closure. We also analyzed hESC line-dependent variability in response to neurotoxicants. We found significant differences that question the reliability of hazard characterization when only one single line is used as a standard.

We showed that hESC-based assays are able to detect specifically developmental toxicity, not caused by cytotoxic effects, and to distinguish effects on morphogenesis and neural differentiation, based on dose-response curves. Using a panel of markers specifically expressed in rosettes, it was possible to distinguish NTD-inducing chemicals acting early during neural induction (CPA or OTA) from those directly impacting neural tube morphogenesis (OTA, VPA and putatively MMF). Notably, OTA, VPA and MMF showed several similarities that might constitute a common pattern. In addition, the two toxicants associated with spina bifida (VPA and MMF) were the most similar by all criteria. Interestingly, while default neurodifferentiation gives rise only to anterior neural cells, the hESC assay was positive for substances associated with closure defects in both the brain (OTA), and the rachis (VPA, MMF). This suggests that developmental mechanisms underlying neural tube formation in different areas are similar enough to avoid the use of specific culture systems to cover a whole range of neurodevelopmental defects. Finally, OTA, which is responsible for both holoprosencephaly and exencephaly, gave rise 
(a)

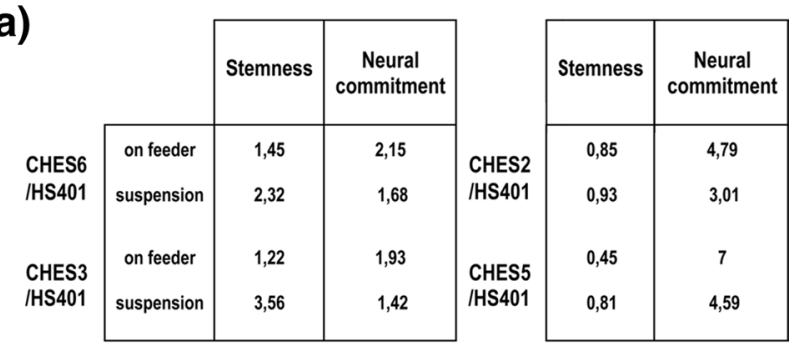

(b)

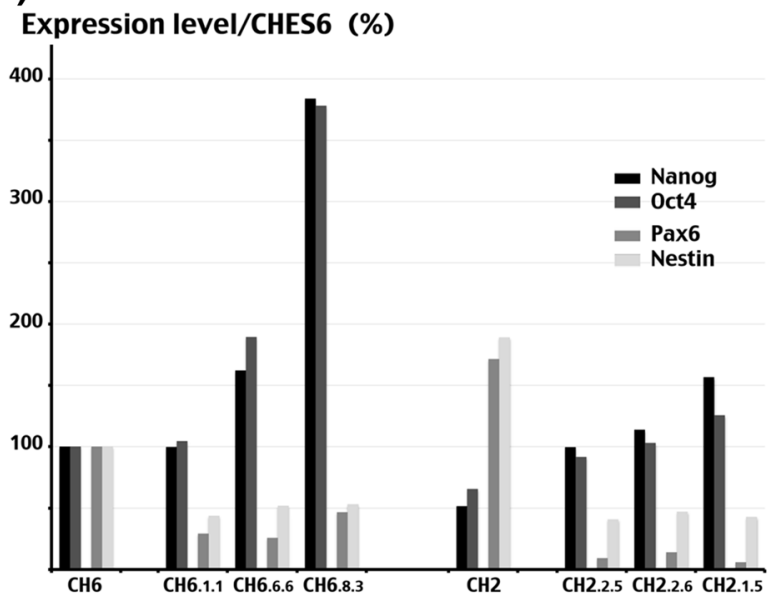

(c)

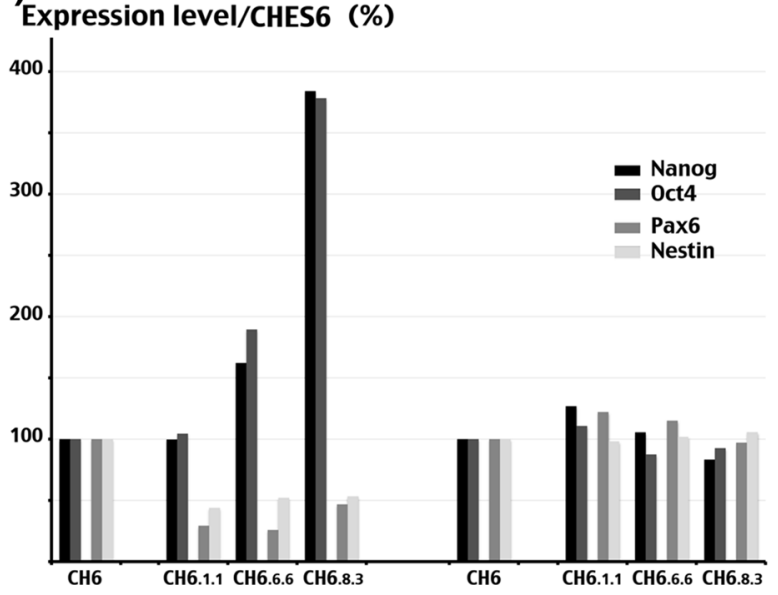

Fig. 5 Relative expression level of stemness and neural commitment markers in cell subpopulations selected from CHES2 and CHES6. a Relative expression of stemness (Oct4) and neural commitment (Pax6) markers, normalized to levels measured with the reference HS401 hESC line, in CHES2, CHES3, CHES5 and CHES6 in maintenance and expansion culture conditions, according to Sterthaus et al. (2014). b Relative expression of stemness (Nanog and Oct4) and neural commitment (Pax6 and Nestin) markers in CHES2, CHES6 and three selected subpopulations of each line, quantified from cultures performed at the same time. For easier comparison, all data are presented relatively to corresponding values measured in CHES6, to which we gave arbitrarily a value of 100. c Relative expression of the same markers in CHES6 and derived subpopulations, presented as in $\mathbf{b}$, before (left) and after expansion in culture (right) to a response profile intermediate between those of toxicants responsible for one single type of defect. This suggests superimposition of two successive effects. An early insult in neural development, with similar consequences on expression of all markers, may precede a more direct effect on rosette formation that would differentially affect the expression of each marker.

We analyzed the reliability of the hESC-based in vitro assay by exposing four different hESC lines to CPA and VPA, which disturb developmental processes at different developmental stages. Although variability of effects with different cell lines has been described (Colleoni et al. 2014), no systematic analysis has been performed so far to evaluate their toxicological relevance. To eliminate the possibility of a differential genetic drift of cell lines in culture, which could interfere with toxicity assessment (Gutbier et al. 2018), we used cell lines derived in one single laboratory, maintained in identical conditions, that have undergone a comparable moderate number of passages in culture (Sterthaus et al. 2014). We nevertheless observed a significant level of variability (up to fivefold) in the responses of the four hESC lines to both compounds. There is thus quite substantial uncertainty in estimating in vitro the toxic potential of chemicals when using a hESC-based assay. However, the arguably relative scarcity of "outlier" individuals might allow a reasonable quantitative estimate of hazard with as few as four cell lines.

The variability in the response to VPA of the four hESC lines correlated with their different stemness/commitment profiles. We have previously shown that hESC lines with a higher tendency to spontaneous commitment exhibited asynchronic differentiation, which increased heterogeneity in the cultures (Sterthaus et al. 2014). Because neural cells at different stages may respond differently to VPA, such an increased heterogeneity could lead to a quantitatively different estimate of hazard. We were unable to eliminate this culture artefact by selecting more homogeneous subpopulations of our hESC lines. This failure is likely due to the described intrinsic heterogeneous and dynamic nature of hESC cell lines in vitro (Espinosa Angarica and Del Sol 2016; Singer et al. 2014).

While line-dependent response variability after treatment with CPA was also observed, it was not correlated with known characteristics of the cell lines. This suggests that the identified inter-line variability may not affect to the same extent compounds acting earlier, at a stage where cell heterogeneity in culture is less pronounced. Notably, response variability after exposure to CPA mainly reflected the divergent behavior of only one cell line compared to the three others, while the "outlier" differed depending on the developmental process analyzed. This variability is reminiscent of individual differences of response to toxicants observed in vivo; variation of sensitivity to toxicants among 
individuals is a well-known phenomenon and risk assessment has to take into account that some people can be under or over-responsive to a particular compound (Dornbos and LaPres 2018; Zeise et al. 2013). Genetic factors may influence the concentration of the active form of the xenobiotic reaching the conceptus by affecting its transport, its metabolism and biotransformation or its ability to cross the placenta (Rogers and Kavlock 2008), and could also directly affect the biological response of the target embryonic cells; this aspect of individual variability has been largely neglected in previous studies.

Altogether, our results based on directed differentiation of hESC show that they have the potential not only to identify developmental toxicants but also to discriminate, confirm and predict developmental alterations. They also emphasize the need to use multiple hESC lines for reliably estimating the dose at which compounds exert a toxic effect in vitro, notwithstanding the currently elusive standardization of hESC lines.

Acknowledgements This research was funded by the Swiss Centre for Applied Human Toxicology (SCAHT, Project 3.1 Neurotox). The authors would like to thank Mrs. Brigitte Schneider for technical assistance. They are grateful to Dr. Rex FitzGerald for comments on the manuscript.

\section{Compliance with ethical standards}

Conflict of interest All authors declare no conflict of interest.

Open Access This article is distributed under the terms of the Creative Commons Attribution 4.0 International License (http://creativeco mmons.org/licenses/by/4.0/), which permits unrestricted use, distribution, and reproduction in any medium, provided you give appropriate credit to the original author(s) and the source, provide a link to the Creative Commons license, and indicate if changes were made.

\section{References}

Balmer NV, Weng MK, Zimmer B et al (2012) Epigenetic changes and disturbed neural development in a human embryonic stem cellbased model relating to the fetal valproate syndrome. Hum Mol Genet 21(18):4104-4114

Bal-Price A, Pistollato F, Sachana M, Bopp SK, Munn S, Worth A (2018) Strategies to improve the regulatory assessment of developmental neurotoxicity (DNT) using in vitro methods. Toxicol Appl Pharmacol 15:15. https://doi.org/10.1016/j.taap.2018.02.008

Baumann J, Gassmann K, Masjosthusmann S et al (2016) Comparative human and rat neurospheres reveal species differences in chemical effects on neurodevelopmental key events. Arch Toxicol 90(6):1415-1427

Cahan P, Daley GQ (2013) Origins and implications of pluripotent stem cell variability and heterogeneity. Nat Rev Mol Cell Biol 14(6):357-368

Colleoni S, Galli C, Gaspar JA et al (2011) Development of a neural teratogenicity test based on human embryonic stem cells: response to retinoic acid exposure. Toxicol Sci 124(2):370-377
Colleoni S, Galli C, Gaspar JA et al (2012) Characterisation of a neural teratogenicity assay based on human ESCs differentiation following exposure to valproic acid. Curr Med Chem 19(35):6065-6071

Colleoni S, Galli C, Gaspar JA et al (2014) A comparative transcriptomic study on the effects of valproic acid on two different hESCs lines in a neural teratogenicity test system. Toxicol Lett 231(1):38-44

Copp AJ, Greene ND (2013) Neural tube defects-disorders of neurulation and related embryonic processes. Wiley Interdiscip Rev Dev Biol 2(2):213-227

Dornbos P, LaPres JJ (2018) Incorporating population-level genetic variability within laboratory models in toxicology: from the individual to the population. Toxicology 395:1-8

Espinosa Angarica V, Del Sol A (2016) Modeling heterogeneity in the pluripotent state: a promising strategy for improving the efficiency and fidelity of stem cell differentiation. Nat Cell Biol 38(8):758-768

Fritsche E, Grandjean P, Crofton KM et al (2018) Consensus statement on the need for innovation, transition and implementation of developmental neurotoxicity (DNT) testing for regulatory purposes. Toxicol Appl Pharmacol 354:3-6

Gassmann K, Abel J, Bothe H et al (2010) Species-specific differential AhR expression protects human neural progenitor cells against developmental neurotoxicity of PAHs. Environ Health Perspect 118(11):1571-1577

Grandjean P, Landrigan PJ (2006) Developmental neurotoxicity of industrial chemicals. Lancet Lond Engl 368(9553):2167-2178

Gutbier S, May P, Berthelot S, Krishna A, Trefzer T, Behbehani M, Efremova L, Delp J, Gstraunthaler G, Waldmann T, Leist M (2018) Major changes of cell function and toxicant sensitivity in cultured cells undergoing mild, quasi-naturalgenetic drift. Arch Toxicol 92:3487-3503

Harrill JA, Freudenrich TM, Robinette BL, Mundy WR (2011) Comparative sensitivity of human and rat neural cultures to chemical-induced inhibition of neurite outgrowth. Toxicol Appl Pharmacol 256(3):268-280

Hoeltzenbein M, Elefant E, Vial T et al (2012) Teratogenicity of mycophenolate confirmed in a prospective study of the European Network of Teratology Information Services. Am J Med Genet Part A 158a(3):588-596

Kelava I, Lancaster MA (2016) Stem cell models of human brain development. Cell Stem Cell 18(6):736-748

Koch P, Opitz T, Steinbeck JA, Ladewig J, Brustle O (2009) A rosette-type, self-renewing human ES cell-derived neural stem cell with potential for in vitro instruction and synaptic integration. Proc Natl Acad Sci USA 106(9):3225-3230

Krug AK, Kolde R, Gaspar JA et al (2013) Human embryonic stem cell-derived test systems for developmental neurotoxicity: a transcriptomics approach. Arch Toxicol 87(1):123-143

Leist M, Hartung T (2013) Inflammatory findings on species extrapolations: humans are definitely no $70-\mathrm{kg}$ mice. Arch Toxicol 87(4):563-567

Lipinski RJ, Godin EA, O’Leary-Moore SK, Parnell SE, Sulik KK (2010) Genesis of teratogen-induced holoprosencephaly in mice. Am J Med Genet Part C Semin Med Genet 154c(1):29-42

Meganathan K, Jagtap S, Wagh V et al (2012) Identification of thalidomide-specific transcriptomics and proteomics signatures during differentiation of human embryonic stem cells. PLoS ONE 7(8):e44228. https://doi.org/10.1371/journal.pone.00442 28

Ornoy A (2006) Neuroteratogens in man: an overview with special emphasis on the teratogenicity of antiepileptic drugs in pregnancy. Reprod Toxicol (Elmsford, NY) 22(2):214-226

Queisser-Luft A, Stolz G, Wiesel A, Schlaefer K, Spranger J (2002) Malformations in newborn: results based on 30,940 infants and 
fetuses from the Mainz congenital birth defect monitoring system (1990-1998). Arch Gynecol Obstet 266(3):163-167

Rogers JM, Kavlock RJ (2008) Developmental toxicology. In: Klaassen CD (ed) Casarett and Doull's toxicology: the basic science of poisons, 7th edn. McGraw Hill, New York, pp 415-452

Schulpen SH, de Jong E, de la Fonteyne LJ, de Klerk A, Piersma AH (2015) Distinct gene expression responses of two anticonvulsant drugs in a novel human embryonic stem cell based neural differentiation assay protocol. Toxicol In Vitro Int J Publ Assoc BIBRA 29(3):449-457

Seok J, Warren HS, Cuenca AG et al (2013) Genomic responses in mouse models poorly mimic human inflammatory diseases. Proc Natl Acad Sci USA 110(9):3507-3512

Shinde V, Klima S, Sureshkumar PS et al (2015) Human pluripotent stem cell based developmental toxicity assays for chemical safety screening and systems biology data generation. J Vis Exp JoVE 100:e52333. https://doi.org/10.3791/52333

Singer ZS, Yong J, Tischler J et al (2014) Dynamic heterogeneity and DNA methylation in embryonic stem cells. Mol Cell 55(2):319-331

Sterthaus O, Feutz AC, Zhang H et al (2014) Gene expression profiles of similarly derived human embryonic stem cell lines correlate with their distinct propensity to exit stemness and their different differentiation behavior in culture. Cell Reprogr 16(3):185-195

Stummann TC, Hareng L, Bremer S (2009) Hazard assessment of methylmercury toxicity to neuronal induction in embryogenesis using human embryonic stem cells. Toxicology 257(3):117-126

Talens-Visconti R, Sanchez-Vera I, Kostic J et al (2011) Neural differentiation from human embryonic stem cells as a tool to study early brain development and the neuroteratogenic effects of ethanol. Stem Cells Dev 20(2):327-339

Waldmann T, Grinberg M, Konig A et al (2017) Stem cell transcriptome responses and corresponding biomarkers that indicate the transition from adaptive responses to cytotoxicity. Chem Res Toxicol 30(4):905-922

Wei X, Sulik KK (1993) Pathogenesis of craniofacial and body wall malformations induced by ochratoxin A in mice. Am J Med Genet 47(6):862-871

Zeise L, Bois FY, Chiu WA, Hattis D, Rusyn I, Guyton KZ (2013) Addressing human variability in next-generation human health risk assessments of environmental chemicals. Environ Health Perspect 121(1):23-31

Zimmer B, Lee G, Balmer NV et al (2012) Evaluation of developmental toxicants and signaling pathways in a functional test based on the migration of human neural crest cells. Environ Health Perspect 120(8):1116-1122

Zimmer B, Pallocca G, Dreser N et al (2014) Profiling of drugs and environmental chemicals for functional impairment of neural crest migration in a novel stem cell-based test battery. Arch Toxicol 88(5):1109-1126

Publisher's Note Springer Nature remains neutral with regard to jurisdictional claims in published maps and institutional affiliations. 